\title{
Desafios e perspectivas para avaliação e melhoria da mobilidade urbana sustentável: um estudo comparativo de municípios brasileiros
}

\author{
Gláucia Maia de Oliveira1 e Antônio Nélson Rodrigues da Silva²
}

\begin{abstract}
Resumo: Este estudo teve como objetivo geral a identificação de desafios e perspectivas para avaliação e melhoria da mobilidade urbana sustentável com base em três análises conduzidas nos seguintes municípios: Belém, Curitiba, Goiânia, Juazeiro do Norte, Uberlândia e Itajubá. A primeira análise foi uma comparação das condições de mobilidade encontradas nas seis cidades selecionadas nas cinco regiões do Brasil, a partir dos resultados obtidos com o Índice de Mobilidade Urbana Sustentável (IMUS). Em seguida, uma estratégia para avaliação e seleção de ações destinadas à promoção da mobilidade sustentável foi aplicada às mesmas cidades e os resultados também comparados. Finalmente, os resultados das duas avaliações foram analisados comparativamente. Embora uma clara correlação entre desempenho e viabilidade tenha sido encontrada, a possibilidade de identificar casos em que isto não ocorre (por exemplo, quando indicadores com baixo desempenho são viáveis de serem melhorados) é também importante. Isto pode indicar uma oportunidade para a melhoria da mobilidade urbana sustentável.
\end{abstract}

Palavras-chave: mobilidade urbana sustentável, indicadores, planejamento da mobilidade.

\begin{abstract}
The general objective of this study was the identification of challenges and perspectives for the evaluation and improvement of sustainable urban mobility, based on three analyses of the following cities: Belém, Curitiba, Goiânia, Juazeiro, Uberlândia, and Itajubá. The first analysis was a comparison of the actual mobility conditions found in six cities selected in the five Brazilian regions, which were assessed with the Index of Sustainable Urban Mobility (I_SUM). Next, a strategy for evaluating and selecting actions for promoting sustainable mobility was applied to the same cities and the results compared. Finally, the outcomes of the two evaluations were comparatively analyzed. Although a clear relationship between performance and viability has been found, the possibility of identifying when performance and viability are not clearly related (for example, when indicators with low performance are highly feasible for improvement), is also important. That condition may provide an opportunity for the improvement of sustainable urban mobility.
\end{abstract}

Keywords: sustainable urban mobility, indicators, mobility planning.

\section{INTRODUÇÃO}

Muitos dos problemas urbanos hoje observados derivam ou têm alguma relação com os sistemas de transporte e circulação. Questões como inadequação da oferta de transporte coletivo, congestionamentos, poluição sonora, poluição do ar e acidentes têm levado a um entendimento generalizado de que a maioria das cidades deve rever suas estratégias a fim de promover padrões sustentáveis de mobilidade. Recentemente foi instituído um importante marco na gestão da política de mobilidade nas cidades brasileiras, através da Lei Federal $n^{0}$ 12.587/2012, conhecida como "Lei da Mobilidade". Esta, além de definir princípios e diretrizes compatíveis com os conceitos de mobilidade urbana sustentável, torna obrigatória a elaboração de Plano de Mobilidade Urbana, integrado e compatível com os respectivos planos diretores ou neles inseridos, para aproximadamente 3.065 municípios (mais de $55 \%$ do total de municípios brasileiros). A lei também concretiza a necessidade de uma sistemática de avaliação, revisão e atualização dos Planos de Mobilidade, tornando imprescindível uma ferramenta de avaliação e controle das

\footnotetext{
${ }^{1}$ Gláucia Maia de Oliveira, Escola de Engenharia de São Carlos Universidade de São Paulo. (gmaia arq@yahoo.com.br)

2 Antônio Nélson Rodrigues da Silva, Escola de Engenharia de São

Carlos - Universidade de São Paulo. (anelson@sc.usp.br)

Manuscrito recebido em 06/03/2014 e aprovado para publicação em 02/03/2015.

Este artigo é parte de TRANSPORTES v. 23, n. 1, 2015. ISSN: 2237-1346 (online). DOI:10.4237/transportes.v23i1.768.
}

condições de mobilidade nos municípios.

Para lidar com tais desafios e com a complexidade do planejamento da mobilidade, tem-se como alternativa o uso de índices e indicadores para o diagnóstico e monitoramento das condições das cidades. O Índice de Mobilidade Urbana Sustentável (IMUS), concebido por Costa (2008), além de ser uma ferramenta de avaliação e controle, se mostra eficiente em orientar as políticas de planejamento e gestão da mobilidade, ao diagnosticar as condições de mobilidade de um município ou região metropolitana. Sua aplicação visando à comparação de resultados sobre as condições de mobilidade entre diferentes municípios ainda necessita, no entanto, ser investigada, tanto para o aprimoramento do índice como para comprovação de sua eficácia enquanto ferramenta de planejamento. Com base nesta premissa, este estudo teve como objetivo geral identificar desafios e perspectivas para avaliação e melhoria da mobilidade urbana sustentável, com base em um conjunto de municípios brasileiros selecionados. A partir deste objetivo geral foram propostos dois específicos: testar o Índice de Mobilidade Urbana Sustentável (IMUS) para comparações entre cidades e testar a relação do IMUS com estratégias de planejamento para melhoria da mobilidade urbana sustentável.

Espera-se que, por meio dos procedimentos propostos, as principais dificuldades que se colocam entre o planejamento da mobilidade e a implantação efetiva de ações de melhoria possam ser reduzidas. Além disso, espera-se que sejam identificados possíveis ajustes no índice para o seu amplo emprego enquanto ferramenta de planejamento, 
principalmente para as etapas de diagnóstico e monitoramento. Uma vez confirmada esta possibilidade de uso para comparação entre os diversos municípios, pode se tornar uma ferramenta de gestão nacional do quadro de mobilidade das diferentes regiões e cidades do Brasil.

O artigo está estruturado da seguinte forma: a seção 2 apresenta uma breve discussão teórica sobre a evolução do conceito de mobilidade urbana sustentável, o uso de índices e indicadores sob este novo paradigma e estudos sobre os principais desafios na implantação de ações para melhoria nas condições de mobilidade. A seção 3 descreve o desenvolvimento e aplicação da metodologia utilizada para a obtenção e sistematização dos dados para análise. O método de análise se apoia na estrutura hierárquica do IMUS e em uma classificação de viabilidade que será detalhada. Discussões sobre os resultados e análises são apresentadas na seção 4, que conduzem às conclusões destacadas na seção que precede as referências bibliográficas.

\section{CONTEXTUALIZAÇÃO TEÓRICA}

Um novo paradigma para o planejamento de transporte vem sendo desenvolvido nas últimas duas décadas. Essa nova visão traz uma abordagem na qual o transporte público, a circulação e o planejamento das atividades urbanas estão associados, dentro do que hoje se conhece como planejamento da mobilidade. Seguindo este avanço, de acordo com Litman e Burwell (2006), há um interesse crescente sobre os conceitos de sustentabilidade e desenvolvimento sustentável. Se o conceito de transporte sustentável é visto como uma extensão do conceito de desenvolvimento sustentável, este pode ser interpretado como o desenvolvimento que proporciona níveis adequados de mobilidade no presente sem comprometer as condições de mobilidade das futuras gerações (GUDMUNDSSON, 2004; RICHARDSON, 2005).

Nesse contexto, novas ferramentas para a prática e o planejamento vêm sendo desenvolvidas para tratar dos problemas de mobilidade sob esse novo paradigma. Entre elas cabe destaque ao aprimoramento de índices e indicadores, os quais são empregados para representar o comportamento de várias funções e elementos que contribuem para a definição do ambiente urbano. De maneira geral, os indicadores inicialmente eram desenvolvidos para a avaliação de impactos econômicos, sociais e ambientais em diferentes cenários. Mais tarde, outros indicadores focaram em aspectos específicos de sustentabilidade, tais como acessibilidade, mobilidade e capacidade ambiental (BLACK et al., 2002; NICOLAS et al., 2003).

A Política Nacional de Mobilidade Urbana, instituída pela Lei Federal n ${ }^{\circ} 12.587 / 2012$, conhecida como "Lei da Mobilidade”, trata do conceito de mobilidade urbana, com a política de transportes associada à de desenvolvimento urbano, e assim orienta governos de nível local. Porém, com relação à efetiva implantação de ações em prol da mobilidade sustentável, o que se observa muitas vezes é que o conceito de mobilidade é associado somente à circulação dos modos motorizados de transporte e a prioridade dada aos motorizados individuais. A etapa de concretização de planos e projetos envolve muitos atores com interesses diversos, e muitas vezes a concretização de um bom planejamento da mobilidade torna-se inviável.

Miranda et al. (2009) buscaram identificar possíveis barreiras para a implantação de um plano de mobilidade contendo os conceitos de sustentabilidade, avaliando a reação de técnicos e gestores de transportes de uma cidade média. Os resultados obtidos levaram a algumas conclusões, entre elas a de que os técnicos e gestores pareciam não dominar integralmente o conceito de mobilidade urbana sustentável, a existência de problemas no arranjo legal vinculado ao Plano Diretor e a outros dispositivos legais já consolidados e a prioridade de ação voltada para o modo motorizado individual.

Ainda nesse sentido, Curtis e Low (2012) analisam como as barreiras institucionais influenciam na transição do planejamento tradicional para o sustentável e como seria possível superá-las, ao estudar três cidades na Austrália. Examinam ainda a teoria da dependência da trajetória (path dependence), que explica porque certas coisas permanecem inalteradas apesar da necessidade de mudanças. Rodrigues da Silva (2013), em sua análise sobre o trabalho de Curtis e Low, comenta sobre a crença dos autores no efeito do discurso de técnicos, políticos e acadêmicos, que para eles desempenham um papel fundamental na mudança de paradigma. Também considera a importância das abordagens utilizadas durante a pesquisa e a possibilidade de uma ampliação do estudo para outras cidades do mundo.

Todos estes pontos foram amplamente discutidos por UN-Habitat (2013), em um documento que, além de discutir os problemas de mobilidade urbana a partir de suas causas, ainda apresenta estratégias para mitigá-los. Estas estratégias são, no entanto, de caráter geral. Seu emprego a situações particulares sempre deve estar sujeito a um processo complementar de avaliação das especificidades locais.

No caso brasileiro, a "Lei da Mobilidade”, além de definir princípios e diretrizes compatíveis com os conceitos de mobilidade urbana sustentável, ao tratar a política de transportes associada à de desenvolvimento urbano, tornou obrigatória a elaboração de Plano de Mobilidade Urbana para aproximadamente 3.065 municípios (mais de $55 \%$ do total de municípios brasileiros). A lei também concretizou a necessidade de uma sistemática de avaliação, revisão e atualização destes planos de mobilidade, tornando imprescindível o uso de ferramentas de avaliação e controle das condições de mobilidade nos municípios. Nesse sentido, destaca-se a importância da coleta e da sistematização de dados para a produção de índices e indicadores, que devem ser utilizados para a orientação das políticas locais de mobilidade e para se obter um panorama nacional das condições de mobilidade do país.

\section{METODOLOGIA}

Com o propósito de atingir os objetivos específicos de testar o Índice de Mobilidade Urbana Sustentável (IMUS) para comparações entre cidades e de testar a relação do IMUS com estratégias de planejamento para melhoria da mobilidade urbana sustentável, foram adotados os seguintes procedimentos: i) apresentação geral dos resultados obtidos em seis cidades selecionadas; ii) análise 
Tabela 1. Dados geográficos das seis cidades selecionadas (em 2010).

\begin{tabular}{llllll}
\hline & $\begin{array}{l}\text { População } \\
\text { urbana } \\
\text { (hab.) }\end{array}$ & $\begin{array}{l}\text { Área urbanizada } \\
(\mathbf{k m}) \text { ) }\end{array}$ & $\begin{array}{l}\text { Densidade } \\
\text { urbana } \\
\text { (hab./km2) }\end{array}$ & $\begin{array}{l}\text { Capital de } \\
\text { Estado }\end{array}$ & $\begin{array}{l}\text { Região Metropolitana } \\
\text { ou Aglomeração Urbana }\end{array}$ \\
\hline Curitiba & 1.587 .315 & 319,47 & $4.968,59$ & sim & RM de Curitiba \\
Belém & 1.272 .354 & 126,80 & $10.034,41$ & sim & RM de Belém \\
Goiânia & 1.085 .806 & 256,82 & $4.227,94$ & sim & RM de Goiânia \\
Uberlândia & 488.982 & 135,35 & $3.612,74$ & não & - \\
Juazeiro do Norte & 202.227 & 12,78 & $15.823,71$ & não & RM do Cariri \\
Itajubá & 76.986 & 11,00 & $7.000,76$ & não & - \\
\hline
\end{tabular}

comparativa dos diagnósticos das condições de mobilidade encontradas pelo cálculo do IMUS; iii) análise comparativa dos valores obtidos através de uma estratégia para avaliação e seleção de ações destinadas à promoção da mobilidade sustentável; e iv) análise comparativa dos valores obtidos nos itens (ii) e (iii). Este estudo se iniciou a partir dos resultados obtidos em trabalhos anteriores da aplicação do Índice de Mobilidade Urbana Sustentável (IMUS) em seis cidades das cinco regiões do Brasil: Belém na região Norte, Curitiba no Sul, Goiânia no Centro-Oeste, Juazeiro do Norte no Nordeste e Uberlândia e Itajubá no Sudeste (por mais detalhes, ver RODRIGUES DA SILVA et al., 2015).

O principal critério na escolha das cidades foi o de que o estudo deveria contemplar pelo menos uma cidade de cada uma das cinco regiões do país. Um fator determinante foi a existência de parceiros comprometidos em realizar a pesquisa em cada uma dessas cidades. Além disso, foi considerado importante que as cidades selecionadas possuíssem características distintas entre si, como população, área urbanizada e papel na dinâmica geográfica. A Tabela 1 resume algumas dessas características para cada cidade selecionada.

O Índice de Mobilidade Urbana (IMUS) é composto por 87 Indicadores, distribuídos em 37 Temas e estes, em 9 Domínios (1. ACESSIBILIDADE, 2. ASPECTOS AMBIENTAIS, 3. ASPECTOS SOCIAIS, 4. AspeCTOS POLÍTICOS, 5. INFRAESTRUTURA DE TRANSPORTES, 6. MODOS NÃO MOTORIZADOS, 7. PlaneJAMENTO INTEGRADO, 8. TRÁFEGO E CIRCULAÇÃO URBANA E 9. SISTEMAS DE TRANSPORTE URBANO). Os Temas e DomínIOS nada mais são do que agrupamentos temáticos que, além de auxiliarem nas análises agregadas, permitem uma redistribuição de pesos conferidos a cada indicador.

O valor global do índice varia em uma escala de 0 (zero) a 1 (um), revelando o diagnóstico das condições da mobilidade urbana sustentável de um município. A estrutura hierárquica do índice, dividida em DomínIOS, Temas

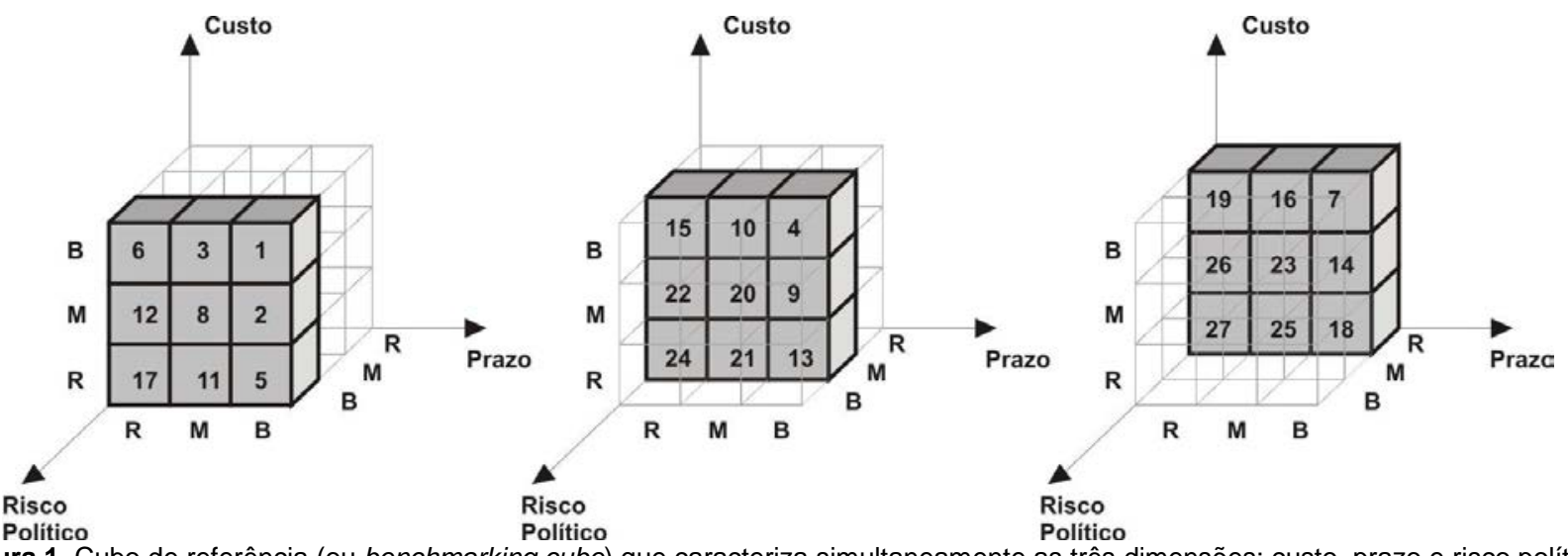

Figura 1. Cubo de referência (ou benchmarking cube) que caracteriza simultaneamente as três dimensões: custo, prazo e risco político Fonte: Mancini (2011) e Indicadores, permite um equilíbrio entre os elementos, sendo que alguns indicadores avaliados com valores muito baixos podem ser compensados por outros com melhores resultados. Além disso, é possível avaliar as condições da mobilidade em aspectos mais específicos, tanto pelos DoMíNIOS como pelos Temas. O IMUS funciona com um sistema de pesos que revela a importância relativa de cada indicador dentro do valor global do índice. No caso de indicadores não calculados por falta de dados, os pesos dos indicadores calculados são redistribuídos dentro do Tema ou do respectivo DOMÍNIO. De forma complementar são calculados os valores do IMUS Superior e IMUS Inferior, que são referências enquanto valores máximos e mínimos possíveis de serem calculados. Para o IMUS Superior, todos os indicadores não calculados são considerados com valor 1,00 (máximo) e para o cálculo do IMUS Inferior os mesmos recebem valor 0,00 (mínimo). Através do cálculo do IMUS Superior e do IMUS Inferior, é possível obter uma escala de variação do IMUS calculado para cada cidade, que torna possível a comparação entre diferentes cidades que não calcularam necessariamente os mesmos indicadores.

A aplicação do IMUS nas seis cidades selecionadas se iniciou com uma análise da disponibilidade e qualidade dos dados necessários para o cálculo dos indicadores em cada cidade (ver RODRIGUES DA SILVA et al., 2015). Após esta análise, os valores do IMUS para cada uma das cidades foram calculados, sendo também calculados os valores do IMUS Superior e IMUS Inferior. Obtiveram-se assim os intervalos para os possíveis valores do IMUS e foi possível comparar os resultados das seis cidades avaliadas. Após a comparação dos valores globais do IMUS, foram analisados os escores obtidos em cada um dos 87 indicadores para cada cidade a fim de se obter informações desagregadas de cada município. Para a análise comparativa aqui desenvolvida, foram selecionados e analisados apenas aqueles indicadores em comum calculados para todas as seis cidades. 
Tabela 2. Blocos de combinações de custo, prazo e risco político no cubo de referência, obtidos conforme a grau de viabilidade. Fonte: Adaptado de Mancini (2011)

\begin{tabular}{|c|c|c|c|c|c|}
\hline 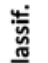 & BLOCOS - CLASSIFICAÇÃO DA VIABILIDADE & \multicolumn{3}{|c|}{ Combinações } & Numeração do cubo de \\
\hline 10 & VIÁVEL em TODOS os quesitos & $\mathbf{B}$ & $\mathbf{B}$ & B & 1 \\
\hline 9 & VIÁVEL em dois quesitos e POUCO VIÁVEL em um & B & B & M & $2,3,4$ \\
\hline 8 & VIÁVEL em dois quesitos e MUITO POUCO VIÁVEL em um & B & B & $\mathbf{R}$ & $5,6,7$ \\
\hline 7 & VIÁVEL em um quesito e POUCO VIÁVEL em outros dois & B & M & M & $8,9,10$ \\
\hline 6 & VIÁVEL em um quesito, POUCO e MUITO POUCO VIÁVEL em outros dois & B & M & $\mathbf{R}$ & $11,12,13,14,15,16$ \\
\hline 5 & VIÁVEL em um quesito e MUITO POUCO VIÁVEL em outros dois & $\mathbf{B}$ & $\mathbf{R}$ & $\mathbf{R}$ & $17,18,19$ \\
\hline 4 & POUCO VIÁVEL em TODOS os quesitos & M & M & M & 20 \\
\hline 3 & POUCO VIÁVEL em dois quesitos e MUITO POUCO VIÁVEL em um & M & M & $\mathbf{R}$ & $21,22,23$ \\
\hline 2 & POUCO VIÁVEL em um quesito e MUITO POUCO VIÁVEL em dois & M & $\mathbf{R}$ & $\mathbf{R}$ & $24,25,26$ \\
\hline 1 & MUITO POUCO VIÁVEL Em TODOS OS quesitos & $\mathbf{R}$ & $\mathbf{R}$ & $\mathbf{R}$ & 27 \\
\hline
\end{tabular}

A partir dos resultados do IMUS de cada cidade, foi utilizado o método para classificação de ações para melhoria das condições de mobilidade de acordo com sua viabilidade, proposto por Mancini (2011) enquanto estratégia de planejamento baseada em cenários. Tal metodologia corresponde à avaliação de três dimensões de viabilidade (Prazo, Custo e Risco Político), simultaneamente analisadas através de um cubo de referência (Figura 1). Esta etapa envolve a aplicação de um questionário a gestores e especialistas de cada cidade, que utiliza uma escala predefinida (com base nos estudos de LIKERT, 1932), com a qual classificam as três dimensões de viabilidade de ações para que cada um dos 87 indicadores anteriormente avaliados atinja o valor máximo de 1,00. Tal avaliação é específica para cada cidade, por partir dos resultados do IMUS anteriormente aplicado. A interpretação dos resultados a partir do cubo de referência é feita através de combinações de viabilidade, variando de "VIÁVEL em todos os quesitos" (obtenção do nível BOM nas três dimensões) até "MUITO POUCO VIÁVEL em todos os quesitos" (obtenção de
RUIM nas três dimensões), conforme a Tabela 2.

Foram aplicados os questionários, para avaliação de viabilidade de ações, a três respondentes em cada cidade, sendo estes especialistas ou gestores municipais, obtendose um total de 18 avaliações. Os resultados foram inicialmente combinados por cidade através do "cubo de referência” e posteriormente, analisados de forma conjunta para as seis cidades. Foram selecionados os indicadores que se enquadraram, em todas as cidades, nas classificações de 7 a 10 do cubo (conforme a Tabela 2), sendo estes os de maior viabilidade, e os classificados de 1 a 4, os de menor viabilidade.

Por último, como forma de se obter informações relativas às estratégias de planejamento associadas ao diagnóstico da mobilidade, foram comparados os resultados obtidos nas etapas anteriores. Para tanto, os dados obtidos nas seis cidades foram agregados em porcentagens de ocorrência em cada um dos indicadores. No caso dos escores do IMUS, os valores foram organizados em cinco classes, incluindo os indicadores não calculados (campo

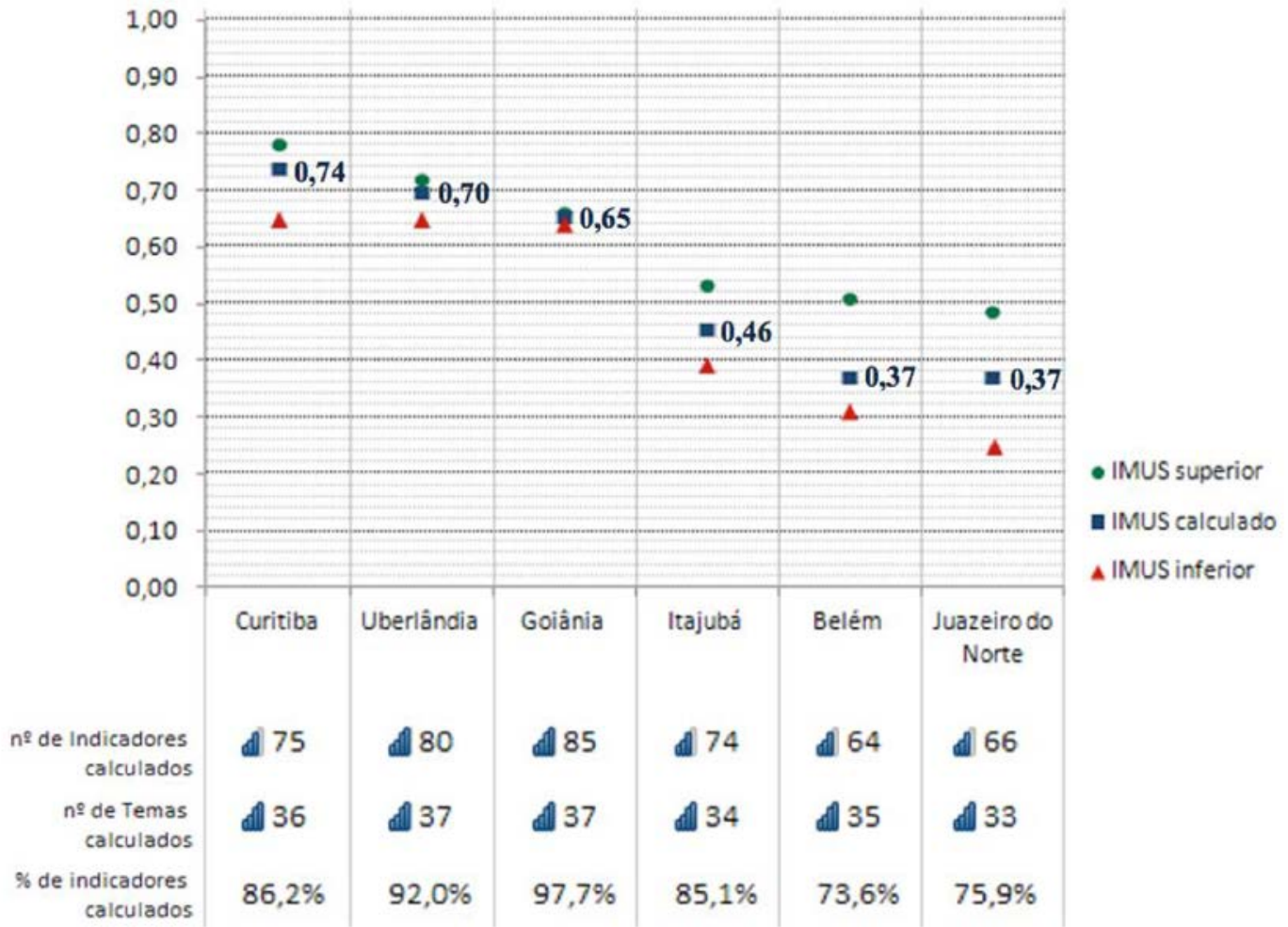

Figura 2. Resultados do cálculo do Índice de Mobilidade Urbana Sustentável para as seis cidades avaliadas 


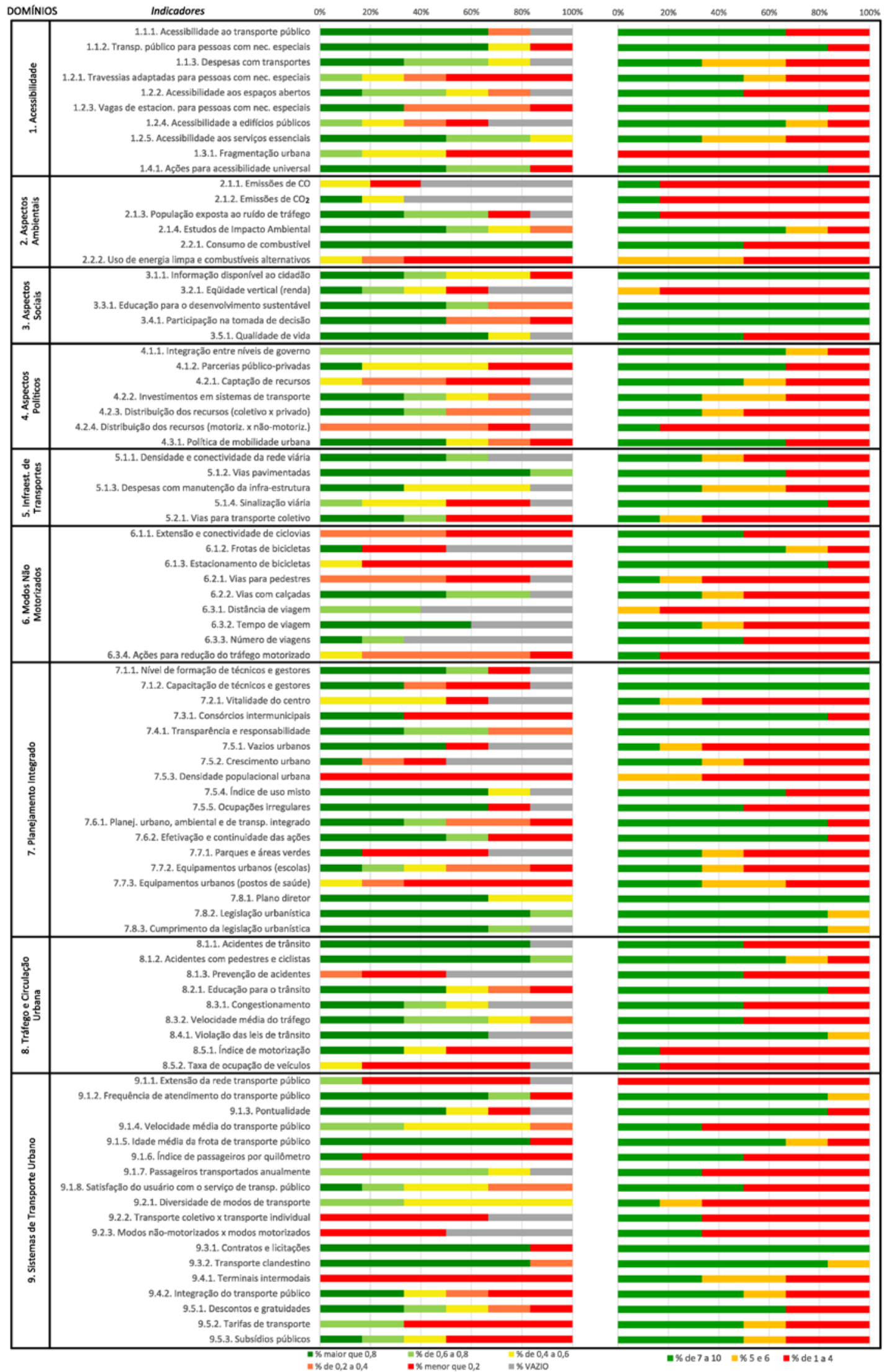

Figura 3. Porcentagens dos escores do IMUS e das classificações de viabilidade obtidos pelo cubo de referência, por indicador (valores agregados das seis cidades)

VAZIO). No caso dos valores obtidos pelo método de classificação de ações de acordo com sua viabilidade, foram organizados em três classes, de acordo com a classificação do “cubo de referência”.

\section{RESULTADOS E DISCUSSÕES}

Esta seção tem início com uma apresentação dos resultados gerais obtidos para as seis cidades estudadas (item 4.1), tanto no que diz respeito aos valores do IMUS e de 
seus indicadores, quanto aos valores resultantes da aplicação da estratégia para avaliação e seleção de ações destinadas à promoção da mobilidade sustentável, conforme o procedimento (i) da metodologia. Esta visão geral será complementada por abordagens mais específicas, de forma a convergir para os objetivos formulados. No item 4.2, são discutidos resultados relativos ao procedimento (ii), que é uma análise comparativa dos diagnósticos da mobilidade nas cidades estudadas. No item 4.3 são colocados os resultados obtidos pela estratégia de avaliação e seleção de ações conforme o procedimento (iii) e, por fim, o item 4.4 aborda os resultados do procedimento (iv), que é uma análise comparativa dos valores obtidos pelos procedimentos (ii) e (iii).

\subsection{Apresentação dos resultados gerais}

Os valores do IMUS encontrados nas seis cidades estudadas estão resumidos na Figura 2. A Figura 3 apresenta valores agregados, das seis cidades estudadas, em porcentagem de ocorrência em cada um dos 87 indicadores que compõem o IMUS, obtidos a partir dos dois métodos adotados. Do lado esquerdo estão os valores que representam o diagnóstico das condições de mobilidade encontradas pelo cálculo do IMUS, classificados em cinco categorias, representadas da cor verde (melhor condição, escore mais alto) à vermelha (pior condição, escore mais baixo), incluindo os indicadores não calculados (VAZIO) representados pela cor cinza. Do lado direito estão os resultados decorrentes da estratégia para avaliação e seleção de ações destinadas à promoção da mobilidade sustentável, organizados em três classes de acordo com a classificação de viabilidade do "cubo de referência”. Os indicadores classificados pelos avaliadores como sendo mais viáveis estão representados pela cor verde e os menos viáveis pela cor vermelha. A análise dos resultados apresentados nesta seção será feita no item 4.2.

\subsection{Análise comparativa dos diagnósticos da mobilidade pelo cálculo do IMUS}

Conforme observado na Figura 2, a partir da comparação dos resultados globais do IMUS para cada cidade, observa-se que Curitiba, Uberlândia e Goiânia apresentaram um desempenho muito melhor do que Itajubá, Belém e Juazeiro do Norte, sendo que mesmo as piores estimativas do primeiro grupo são melhores do que as melhores estimativas do segundo grupo. Nota-se que aquelas cidades com maior quantidade de indicadores não calculados possuem uma maior variação e, portanto, menor precisão no resultado final do índice.

A partir da análise dos indicadores calculados, foi possível observar que 46 do total de 87 indicadores que compõem o IMUS foram calculados para as seis cidades (Figura 4). Assim, do total de 37 Temas, 7 tiveram pelo menos um indicador não calculado por alguma das seis cidades.

Alguns indicadores se destacaram por resultados muito ruins na maioria das cidades. Se por um lado isto pode revelar problemas em comum nas seis cidades, por outro, pode indicar a necessidade de revisão do próprio método de cálculo ou dos valores de referência. Um exemplo é o indicador 6.1.3. Estacionamento de bicicletas que, com exceção de Goiânia, em todas as cidades obteve escore 0,00. A baixa avaliação deste indicador, que mede a integração modal entre transporte público e bicicletas, pode evidenciar o baixo investimento dos governos locais neste tipo de infraestrutura. Por outro lado, tal resultado indica a possibilidade de adequação do indicador, que poderia também medir a existência de paraciclos ou bicicletários em vias, prédios e espaços públicos. Consideraria assim, não apenas a intermodalidade, mas também a existência de ações voltadas à criação ou manutenção de polos geradores de viagens sustentáveis.

Todas as seis cidades obtiveram escores muito baixos no indicador 7.5.3. Densidade populacional urbana. Isto ocorreu inclusive em Juazeiro do Norte e Belém, que possuem altas densidades demográficas (respectivamente, 15.824 e 10.034 hab. $/ \mathrm{km}^{2}$ ), se comparadas às outras cidades analisadas. Tal fato indica a necessidade de revisão dos valores de referência do indicador, que tem como objetivo avaliar a concentração da população em áreas bem servidas de infraestrutura urbana, além do controle da expansão urbana. Isto já havia sido observado por Miranda (2010) em Curitiba, exemplo de cidade desenvolvida nos moldes do Transit Oriented Development (como discutido em UN-Habitat, 2013). A cidade, que possui alto coeficiente de ocupação nas áreas próximas aos corredores de transporte público e menores densidades fora destes, foi prejudicada na avaliação deste indicador, recebendo escore 0,00 .

Outros indicadores se destacaram por obter boa avaliação em comum na maioria das cidades avaliadas, revelando também pontos críticos específicos em algumas delas. Os municípios analisados foram muito bem avaliados no indicador 9.1.5. Idade média da frota de transporte público, com exceção de Juazeiro do Norte, que obteve escore 0,00. O mesmo ocorre com os indicadores 9.3.1. Contratos e licitações e 9.3.2. Transporte clandestino, que indicam que os municípios foram muito bem avaliados com relação à regularização dos contratos de operação de serviços de transporte público e com a inexistência de transporte clandestino, recebendo todos escore máximo (com exceção de Belém, que foi muito mal avaliada em ambos indicadores). Foi possível destacar ainda Juazeiro do Norte, que foi muito mal avaliada nos indicadores relativos à acessibilidade para pessoas com deficiência, obtendo escore 0,00 nos quatro indicadores.

A análise dos escores por indicador evidenciou alguns resultados em comum pela categoria DomínIOs do IMUS. Enquanto Curitiba, Goiânia e Uberlândia se destacaram por terem seus indicadores muito bem avaliados no domínio 3. AsPECTOS SOCIAIS, todas as cidades obtiveram escores baixos no domínio 6. MODOS NÃO MOTORIZADOS, o que evidencia a falta de prioridade que os governos locais dão a este modo de transporte. Dentro do domínio 2. Aspectos AMBIENTAIS, enquanto o indicador 2.2.1. Consumo de combustível foi muito bem avaliado em todas as cidades, o indicador 2.2.2. Uso de energia limpa e combustíveis alternativos, que mede o uso destes combustíveis no transporte público, foi mal avaliado em todas elas, obtendo escore 0,00 em quatro das cidades. Isso mostra que, apesar do consumo de gasolina por habitante ser baixo, não existe o incentivo para uso de combustíveis 


\begin{tabular}{|c|c|c|c|c|c|c|c|c|}
\hline DOMÍNIOS & TEMAS & INDICADORES & 炼 & $\begin{array}{l}E \\
\frac{E}{\Phi} \\
\Phi\end{array}$ & $\begin{array}{l}\frac{\pi}{\mathbb{E}} \\
\frac{\pi}{0} \\
0\end{array}$ & $\begin{array}{l}\frac{10}{7} \\
\frac{5}{2} \\
\frac{60}{2} \\
5 \\
5\end{array}$ & 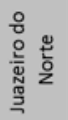 & 粱 \\
\hline \multirow{6}{*}{ 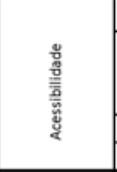 } & 1.1 & 1.1.2. Transporte público para pessoas com necessidades especiais & 1,00 & 0,42 & 1,00 & 1,00 & 0,00 & 1,00 \\
\hline & \multirow{3}{*}{1.2} & 1.2.1. Travessias adaptadas para pessoas com necessidades especiais & 0,60 & 0,15 & 0,10 & 0,45 & 0,00 & 0,23 \\
\hline & & 1.2.3. Vagas de estacionamento para pessoas com necessidades especiais & 0,20 & 0,20 & 1,00 & 0,80 & 0,00 & 0,20 \\
\hline & & 1.2.5. Acessibilidade aos serviços essenciais & 0,85 & 0,72 & 0,52 & 0,80 & 0,87 & 0,61 \\
\hline & 1.3 & 1.3.1. Fragmentação urbana & 0,00 & 0,40 & 0,00 & 0,00 & 0,70 & 0,55 \\
\hline & 1.4 & 1.4.1. Açōes para acessibilidade universal & 1,00 & 0,75 & 1,00 & 1,00 & 0,00 & 0,75 \\
\hline \multirow{3}{*}{ 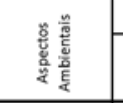 } & 2.1 & 2.1.4. Estudos de Impacto Ambiental & 1,00 & 0,50 & 1,00 & 1,00 & 0,75 & 0,25 \\
\hline & \multirow{2}{*}{2.2} & 2.2.1. Consumo de combustivel & 0,99 & 1,00 & 0,83 & 0,94 & 1,00 & 0,81 \\
\hline & & 2.2.2. Uso de energia limpa e combustiveis alternativos & 0,35 & 0,00 & 0,00 & 0,52 & 0,00 & 0,00 \\
\hline \multirow{3}{*}{ 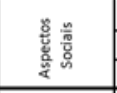 } & 3.1 & 3.1.1. Informaçăo disponivel ao cidadão & 1,00 & 0,50 & 0,75 & 1,00 & 0,00 & 0,50 \\
\hline & 3.3 & 3.3.1. Educação para o des envolvimento sustentável & 0,75 & 0,25 & 1,00 & 1,00 & 1,00 & 0,25 \\
\hline & 3.4 & 3.4.1. Participação na tomada de decisão & 1,00 & 0,33 & 1,00 & 1,00 & 0,33 & 0,00 \\
\hline \multirow{3}{*}{ 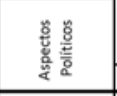 } & \multirow{2}{*}{4.2} & 4.1.1. Integração entre niveis de governo & 0,75 & 0,75 & 0,75 & 0,75 & 0,75 & 0,75 \\
\hline & & 4.1.2. Parcerias público-privadas & 1,00 & 0,00 & 0,50 & 0,50 & 0,00 & 0,50 \\
\hline & 4.3 & 4.3.1. Politica de mobilidade urbana & 1,00 & 0,50 & 1,00 & 1,00 & 0,25 & 0,00 \\
\hline \multirow{2}{*}{ 总总 } & 5.1 & 5.1.2. Vias pavimentadas & 0,89 & 0,64 & 0,96 & 0,91 & 0,80 & 0,92 \\
\hline & 5.2 & 5.2.1. Vias para transporte coletivo & 0,90 & 0,00 & 0,66 & 0,80 & 0,00 & 0,00 \\
\hline \multirow{3}{*}{ 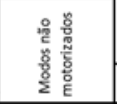 } & \multirow{2}{*}{6.1} & 6.1.1. Extensão e conectividade de ciclovias & 0,25 & 0,25 & 0,25 & 0,00 & 0,00 & 0,00 \\
\hline & & 6.1.3. Estacionamento de bicicletas & 0,00 & 0,00 & 0,45 & 0,00 & 0,00 & 0,00 \\
\hline & 6.3 & 6.3.4. Açōes para redução do tráfego motorizado & 0,25 & 0,00 & 0,50 & 0,25 & 0,25 & 0,25 \\
\hline \multirow{9}{*}{ 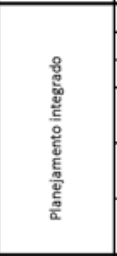 } & 7.3 & 7.3.1. Consórcios intermunicipais & 1,00 & 0,00 & 1,00 & 0,00 & 0,00 & 0,00 \\
\hline & 7.4 & 7.4.1. Transparência e responsabilidade & 1,00 & 0,75 & 0,75 & 1,00 & 0,25 & 0,25 \\
\hline & 7.5 & 7.5.3. Densidade populacional urbana & 0,00 & 0,11 & 0,00 & 0,00 & 0,00 & 0,06 \\
\hline & \multirow{2}{*}{7.6} & 7.6.1. Planejamento urbano, ambiental e de transporte integrado & 1,00 & 0,33 & 0,66 & 1,00 & 0,00 & 0,33 \\
\hline & & 7.6.2. Efetivação e continuidade das açöes & 1,00 & 0,00 & 1,00 & 1,00 & 0,00 & 0,75 \\
\hline & \multirow{2}{*}{7.7} & 7.7.2. Equipamentos urbanos (escolas) & 0,10 & 0,27 & 0,54 & 0,25 & 0,88 & 0,70 \\
\hline & & 7.7.3. Equipamentos urbanos (postos de saúde) & 0,00 & 0,00 & 0,02 & 0,00 & 0,36 & 0,47 \\
\hline & \multirow{2}{*}{7.8} & 7.8.1. Plano diretor & 1,00 & 1,00 & 1,00 & 1,00 & 0,50 & 0,50 \\
\hline & & 7.8.2. Legislaçăo urbanistica & 1,00 & 1,00 & 1,00 & 1,00 & 0,70 & 1,00 \\
\hline \multirow{4}{*}{ 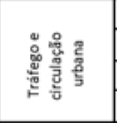 } & 8.1 & 8.1.2. Acidentes com pedestres e ciclistas & 1,00 & 0,63 & 0,92 & 1,00 & 1,00 & 0,95 \\
\hline & 8.2 & 8.2.1. Educação para o trânsito & 1,00 & 0,25 & 0,42 & 0,82 & 0,00 & 1,00 \\
\hline & 8.3 & 8.3.2. Velocidade média do tráfego & 0,38 & 0,54 & 1,00 & 0,74 & 0,68 & 1,00 \\
\hline & 8.5 & 8.5.1. Índice de motorização & 0,00 & 1,00 & 0,00 & 0,00 & 0,91 & 0,41 \\
\hline \multirow{13}{*}{ 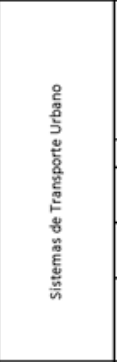 } & \multirow{5}{*}{9.1} & 9.1.2. Frequência de atendimento do transporte público & 1,00 & 1,00 & 0,61 & 1,00 & 1,00 & 0,00 \\
\hline & & 9.1.4. Velocidade média do transporte público & 0,45 & 0,48 & 0,29 & 0,50 & 0,65 & 0,75 \\
\hline & & 9.1.5. Idade média da frota de transporte público & 0,83 & 1,00 & 1,00 & 1,00 & 0,00 & 1,00 \\
\hline & & 9.1.6. Índice de passageiros por quilômetro & 1,00 & 0,15 & 0,03 & 0,00 & 0,00 & 0,00 \\
\hline & & 9.1.8. Satisfaçăo do usuário com o serviço de transporte público & 0,59 & 0,31 & 0,27 & 0,80 & 0,74 & 0,50 \\
\hline & 9.2 & 9.2.1. Diversidade de modos de transporte & 0,50 & 0,75 & 0,50 & 0,50 & 0,75 & 0,50 \\
\hline & \multirow{2}{*}{9.3} & 9.3.1. Contratos e licitações & 1,00 & 0,00 & 1,00 & 1,00 & 1,00 & 1,00 \\
\hline & & 9.3.2. Transporte clandestino & 1,00 & 0,25 & 1,00 & 1,00 & 1,00 & 1,00 \\
\hline & \multirow{2}{*}{9.4} & 9.4.1. Terminais intermodais & 0,00 & 0,00 & 0,00 & 0,00 & 0,00 & 0,00 \\
\hline & & 9.4.2. Integração do transporte público & 1,00 & 0,25 & 1,00 & 0,50 & 0,00 & 0,00 \\
\hline & \multirow{3}{*}{9.5} & 9.5.1. Descontos e gratuidades & 1,00 & 0,05 & 0,67 & 0,82 & 0,31 & 0,46 \\
\hline & & 9.5.2. Tarifas de transporte & 0,66 & 0,00 & 0,00 & 0,00 & 0,66 & 0,00 \\
\hline & & 9.5.3. Subsídios públicos & 0,75 & 0,00 & 0,50 & 0,00 & 0,00 & 1,00 \\
\hline 09 Domínios & 30 Temas & 46 indicadores & & & & & & \\
\hline
\end{tabular}

Figura 4. Valores dos escores obtidos para os 46 indicadores em comum (calculados para as seis cidades), por cidade

renováveis e menos poluentes no transporte público das cidades analisadas.

\subsection{Análise comparativa dos valores obtidos pela estratégia de avaliação e seleção de ações destinadas à promoção da mobilidade sustentável}

Conforme se verifica na Figura 5, dos questionários sobre viabilidade de ações aplicados aos avaliadores, destacam-se dez indicadores com classificação em comum nas seis cidades.

Oito dos indicadores destacados (3.1.1 Informação disponível ao cidadão, 3.3.1 Educação para o desenvolvimento sustentável, 3.4.1 Participação na tomada de decisão, 7.1.1 Nível de formação de técnicos e gestores, 7.1.2 Capacitação de técnicos e gestores, 7.4.1 Transparência e responsabilidade, 7.8.1 Plano diretor e 9.3.1 Contratos $e$ licitações) foram considerados com alta viabilidade de ações para melhoria e apenas dois deles (1.3.1 Fragmentação urbana e 9.1.1 Extensão da rede transporte público) foram classificados com baixa viabilidade. Os temas 7.1 Capacitação de gestores e 7.4 Transparência do processo de planejamento, que compõem o domínio 7. PlAnEJAMENTO INTEGRADO, foram classificados como sendo de alta viabilidade de ações em todas as cidades. O mesmo ocorre com os temas 3.1 Apoio ao cidadão, 3.3 Educação e cidadania e 3.4 Participação popular que compõem o domínio 3. AsPECTOS SOCIAIS. Tal resultado apresenta um importante caminho a ser seguido pelos gestores municipais por serem ações de baixo risco político, baixo custo e de rápida execução. Com o tema 1.3 Barreiras físicas ocorre o oposto, sendo este totalmente classificado como muito inviável, o que reflete a grande dificuldade em se fazer alterações estruturais no tecido urbano já consolidado.

\subsection{Análise comparativa dos valores obtidos do diagnóstico IMUS e classificação de viabilidade}

Conforme pôde ser observado na Figura 3, ao se comparar os resultados agregados de diferentes cidades, os indicadores mal avaliados no IMUS, ou seja, de baixo desempenho, em sua maioria foram também classificados como de baixa viabilidade para serem melhorados, como por exemplo os indicadores 7.5.3 Densidade populacional urbana e 9.1.1 Extensão da rede de transporte público. Da mesma maneira, aqueles indicadores que foram melhor avaliados (bom desempenho), também foram classificados 


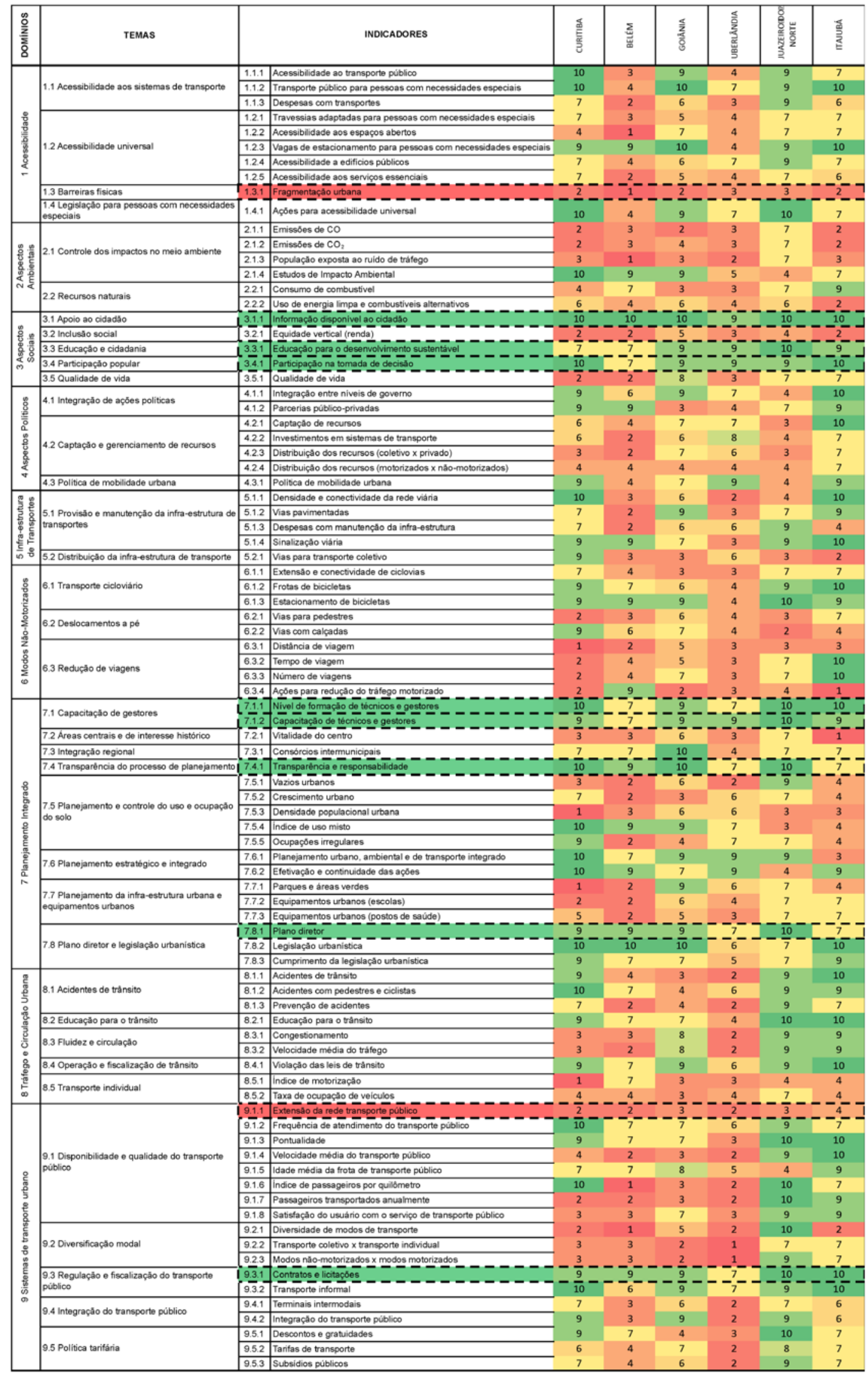

Figura 5. Classificação dos 87 indicadores quanto à viabilidade de ações, considerando custo, prazo e risco político, a partir da análise do "cubo de referência".

como de alta viabilidade. Tal relação ocorre, tanto devido à facilidade de um indicador com escore alto atingir 0 escore máximo, tanto pela facilidade de melhorar políticas que já estão implantadas, sendo os custos apenas de manutenção destas políticas.
Outro resultado foi a correlação entre aqueles indicadores não avaliados por falta de dados e a baixa viabilidade de melhoria dos mesmos, como, por exemplo, os indicadores 6.3.1, 6.3.2 e 6.3.3 (Distância, Tempo de Número de viagens) e 2.1.1 e 2.1.2 (Emissões de $\mathrm{CO}$ e de $\mathrm{CO} 2$ ). Tal fato indica a grande dificuldade de alguns mu- 
nicípios na coleta e organização de importantes dados, muitas vezes por falta de estrutura institucional e de pessoal. Se tais governos locais não conseguem mensurar alguns dados para um diagnóstico mais preciso, não conseguem também planejar melhorias e estipular metas a serem atingidas.

Considerando que tais correlações predominam, se evidenciam alguns desvios, como os indicadores 6.1.2 e 6.1.3 (Frota e Estacionamentos de bicicletas) que, apesar de mal avaliados, possuem alta viabilidade de serem melhorados. Tal resultado indica ações imediatas de melhoria de determinados aspectos críticos que devem ser tomadas, sendo estas de baixo custo e risco político.

\section{CONCLUSÕES}

Com base nos resultados do IMUS obtidos para as seis cidades analisadas, pode-se concluir que os diferentes contextos afetam diretamente as condições de mobilidade de cada cidade. De modo geral, as cidades melhor avaliadas se encontram nas regiões mais ricas do país. Também é possível perceber que as cidades menores obtiveram resultados piores na avaliação. Tais observações podem levar a conclusões sobre as diferentes realidades regionais do país e também as especificidades de cada município avaliado, podendo assim auxiliar no planejamento, não só na esfera municipal, mas também nas esferas estadual e federal.

A partir da análise dos resultados por indicadores, dos 87 indicadores que compõem o IMUS, apenas 46 foram calculados pelas seis cidades, o que representa $53 \%$ do total. Tal resultado parece sugerir que uma revisão do método de cálculo do IMUS pode ser necessária, tanto pela quantidade de indicadores que pode ser excessiva, quanto pelo método de cálculo de alguns dos indicadores. No segundo caso, ou o cálculo se mostra demasiado complexo ou exige dados difíceis de serem coletados, o que levou à impossibilidade de cálculo de muitos dos indicadores. Pode-se concluir ainda que, em alguns casos, os valores de referência originalmente estipulados por Costa (2008) não estão adequados para uma ampla aplicação em distintas cidades, como foi o caso de Densidade Populacional e Fragmentação Urbana, o que pode levar a avaliações não condizentes com a realidade local. Por outro lado, um aspecto positivo da aplicação do IMUS em distintas cidades foi a possibilidade de observar pontos críticos em comum e também características alinhadas ao conceito de mobilidade urbana sustentável em vários municípios, sendo possível ainda destacar eventuais desvios. É importante ressaltar que, pela complexidade de se comparar cidades de características tão distintas, um desafio que se coloca é a definição de um grupo de indicadores centrais que possam ser aplicados em qualquer contexto urbano, sem consideráveis distorções em seus resultados.

Com relação à análise de viabilidade de ações nas dimensões prazo, custo e risco político, os indicadores que foram classificados pelos avaliadores de forma semelhante nas seis cidades representam menos de $15 \%$ do total de 87 indicadores que compõem o IMUS. Assim, tais discrepâncias na classificação de viabilidade, revelaram cidades mais otimistas, como Juazeiro do Norte e Itajubá, e cidades mais pessimistas, como Belém e Uberlândia. Esta última, apesar de ser bem avaliada pelo IMUS, considera muitas ações de melhoria como inviáveis. Desse modo, a partir da análise do "cubo de referência”, pode-se concluir que, ou não há uma considerável concordância entre as seis cidades analisadas com relação à viabilidade de se executar medidas visando à melhoria das condições da mobilidade urbana, ou a amostra de três avaliadores em cada cidade é insuficiente para uma conclusão definitiva.

Conforme desenvolvido por Mancini (2011), uma análise comparativa entre o diagnóstico da mobilidade através do IMUS e a viabilidade de ações em uma cidade específica pode trazer resultados importantes no sentido de caracterizar os desafios e perspectivas para atingir padrões sustentáveis de mobilidade urbana, tais como identificar situações críticas e ações mitigadoras mais ou menos exequíveis. Ao se comparar os resultados agregados de diferentes cidades, uma forte relação entre bom desempenho e alta viabilidade se evidencia, sendo que a maioria dos indicadores mal avaliados no IMUS foram também classificados como de baixa viabilidade para serem melhorados. Isso pode ter ocorrido pelo fato de que o desempenho dos indicadores foi avaliado de forma quantitativa, enquanto que a viabilidade foi avaliada de forma qualitativa. Independente disto, o ponto mais importante desta avaliação talvez seja a possibilidade de identificar os casos em que isto não ocorre, como alguns indicadores que embora críticos, são ao mesmo tempo altamente viáveis de serem melhorados.

\section{REFERÊNCIAS}

ASSUNÇÃO, M. A. (2012) Indicadores de Mobilidade Urbana Sustentável para a Cidade de Uberlândia, MG. Dissertação (Mestrado). Faculdade de Engenharia Civil, Universidade Federal de Uberlândia, Uberlândia. http://www.ppgec.feciv.ufu.br/node/329.

AZEVEDO FILHO, M. A. N. (2012) Análise do Processo de Planejamento dos Transportes como Contribuição para a Mobilidade Urbana sustentável. Tese (Doutorado). Escola de Engenharia de São Carlos, Universidade de São Paulo, São Carlos. http://www.teses.usp.br/teses/disponiveis/18/18144/tde-1112201 2-091904/.

BLACK, J. A.; PAEZ, A.; SUTHANAYA, P.A. (2002) Sustainable Urban Transportation: Performance Indicators and Some Analytical Approaches. Journal of Urban Planning and Development, v. 128, n. 4, p. 184-209. DOI: 10.1061/(ASCE)0733 9488(2002)128:4(184).

BRASIL. (2012) Lei no 12.587, de 03 de janeiro de 2012. Institui as Diretrizes da Política Nacional de Mobilidade Urbana e dá outras providências. Brasília. http://www.planalto.gov.br/cci vil_03/_ato20112014/2012/lei/l12587.htm.

COSTA, M. S. (2008) Um Índice de Mobilidade Urbana Sustentável. Tese (Doutorado). Escola de Engenharia de São Carlos, Universidade de São Paulo, São Carlos. http://www.teses.usp.br/teses/disponiveis/18/18144/tde01112008 $-200521 /$.

CURTIS, C. e LOW, N. (2012) Institutional Barriers to Sustainable Transport. Ashgate, Farnham, Surrey.

GUDMUNDSSON, H. (2001) Indicators and Performance Measures for Transportation, Environment and Sustainability in North America. Relatório do German Marshall Fund Fellowship 2000. Individual Study Tour October 2000. Research Notes n. 
148.http://www2.dmu.dk/1_Viden/2_Publikationer/3_abraporter/ rapporter/AR148.pdf.

GUDMUNDSSON, H. (2004) Sustainable Transport and Performance Indicators, In: HESTER, R.E. \& HARRISON, R.M. (eds), Transport and the Environment - Issues in Environmental Science and Technology, n. 20, Royal Society of Chemistry, Cambridge-UK, p. 35-63. http://orbit.dtu.dk/fedora/objects/orbit:22485/datastreams/file_98 1cc12d-1a6a-4a0c-94b6-6ecf0e650669/content.

IPEA. (2011) A Nova Lei de Diretrizes da Política Nacional de Mobilidade Urbana. (Comunicados do IPEA). Instituto de Pesquisa Econômica Aplicada, Brasília. http://www.ipea.gov.br/portal/images/stories/PDFs/comunicado/ 120106_comunicadoipea128.pdf.

LIKERT, R. A. (1932) A Technique for the Measurement of Attitudes. Archives of Psychology, v. 140, n. 1, p. 1-55. http://www.voteview.com/Likert_1932.pdf.

LITMAN, T. e BURWELL, D. (2006) Issues in Sustainable Transportation. International Journal of Global Environmental Issues, v. 6, n. 4, p. 331-347. http://www.vtpi.org/sus_iss.pdf.

LITMAN, T. (2011) Well measured - Developing Indicators for Sustainable and Livable Transport Planning. Victoria Transport Policy Institute. Victoria. http://www.vtpi.org/wellmeas.pdf.

MANCINI, M. T. (2011) Planejamento Urbano Baseado em Cenários de Mobilidade Sustentável. Dissertação (Mestrado). Escola de Engenharia de São Carlos da Universidade de São Paulo, São Carlos. http://www.teses.usp.br/teses/disponiveis/18/18144/tde14062011 $-174919 /$.

MIRANDA, H. F. (2010) Mobilidade Urbana Sustentável e o Caso de Curitiba. Dissertação (Mestrado). Escola de Engenharia de São Carlos, Universidade de São Paulo, São Carlos. http://www.teses.usp.br/teses/disponiveis/18/18144/tde03052011 $-103404 /$

MIRANDA, H. F. e RODRIGUES DA SILVA, A. N. (2012) Benchmarking Sustainable Urban Mobility: The Case of Curitiba, Brazil. Transport Policy, v. 21, p. 141-151. DOI:10.1016/j.tranpol.2012.03.009.

MIRANDA, H. F.; MANCINI, M. T.; AZEVEDO FILHO, M. A. N. D.; ALVES, V. F. B.; RODRIGUES DA SILVA, A. N. (2009) Barreiras para a Implantação de Planos de Mobilidade. In: XXIII ANPET - Congresso de Pesquisa e Ensino em Transportes, Vitória. ANPET, p. 1-12.

MORAIS, T. C. (2012) Avaliação e Seleção de Alternativas para a Promoção da Mobilidade Urbana Sustentável - O Caso de Anápolis, Goiás. Dissertação (Mestrado). Escola de Engenharia de São Carlos, Universidade de São Paulo, São Carlos. http://www.teses.usp.br/teses/disponiveis/18/18144/tde07022013 $-164654 /$.

NICOLAS, J. P.; POCHET, P.; POIMBOEUF, H. (2003) Towards Sustainable Mobility Indicators: Application to the Lyons Conurbation. Transport Policy, v. 10, n. 3, p. 197-208. DOI: 10.1016/S0967-070X(03)00021-0.

RICHARDSON, B. C. (2005) Sustainable Transport: Analysis Frameworks. Journal of Transport Geography, n. 13, 29-39. DOI: 10.1016/j.jtrangeo.2004.11.005.
RODRIGUES DA SILVA, A. N.; AZEVEDO FILHO, M. A. N.; MACEDO, M. H.; SORRATINI, J. A.; SILVA, A. F.; LIMA, J. P. E PINHEIRO, A. M. G. S. (2015) A Comparative Evaluation of Mobility Conditions in Selected Cities of the Five Brazilian Regions. Transport Policy, v. 37, n. 1, p. 147-156. DOI: 10.1016/j.tranpol.2014.10.017.

RODRIGUES DA SILVA, A. N. (2013) Institutional Barriers to Sustainable Transport, C. Curtis, N. Low. Journal of Transport Geography. (Book Review). DOI: 10.1016/j.jtrangeo.2013.04. 004 .

RODRIGUES DA SILVA, A. N.; COSTA, M. S.; MACEDO, M. H. (2008) Multiple Views of Sustainable Urban Mobility The Case of Brazil. Transport Policy, v. 15, n. 6, p. 350-360. DOI:10.1016/j.tranpol.2008.12.003.

UN-HABITAT. (2013) Planning and Design for Sustainable Urban Mobility: Global Report on Human Settlements 2013, United Nations Human Settlements Programme. Nairobi: UNHabitat, Abingdon [etc.]: Earthscan from Routledge. http://unhabitat.org/planning-and-design-for-sustainable-urbanmobility-global-report-on-human-settlements-2013/. 